\title{
Construction of Fuzzy Relation by Closure Systems
}

\author{
Vladimír Janiš ${ }^{1}$, Magdalena Renčova ${ }^{1, \star}$, Branimir Šešelja ${ }^{2}$, \\ and Andreja Tepavčević ${ }^{2, \star \star}$ \\ 1 Department of Mathematics, Faculty of Natural Sciences, Matej Bel University, \\ SK-974 01 Banská Bystrica, Slovak Republic \\ 2 Department of Mathematics and Informatics, University of Novi Sad, Serbia
}

\begin{abstract}
Starting with a collection of closure systems each of which is associated to an element of a given set $X$, we construct a lattice $L$ and an $L$-fuzzy relation on $X$, such that its fuzzy blocks are precisely the given closure systems.
\end{abstract}

AMS Mathematics Subject Classification (2000): primary 03B52, 03E72; secondary 06A15.

Keywords and phrases: lattice-valued fuzzy set, lattice-valued fuzzy relation, block, cut.

\section{Introduction}

As it is known in the classical theory of relations, a block of a binary relation on a domain $X$ is its subset associated to an element $x$ of $X$ : it contains all elements from the domain related to this particular element. The most known are blocks of an equivalence relation which split the domain into a quotient set; for an ordering relation, a block of an element is a principal filter generated by this element etc. In many applications of binary relations blocks are actually used; that is why they are very important. In addition, it is easy to (re)construct the relation if all blocks are known.

In the case of fuzzy (lattice valued) relations, the situation is similar, though much more complicated. Blocks of a fuzzy relation are (defined analogously as in the crisp case) fuzzy sets on the same domain. For particular fuzzy relations, blocks have been widely investigated by many authors, though under different names. Gottwald (see his book [5]) introduces a kind of blocks using the name full image of the fuzzy set under a fuzzy relation (these correspond to Zadeh's conditional fuzzy sets). In the book [6] by Klir and Yuan, one can find blocks of a fuzzy order,

\footnotetext{
* The research supported by grant no. 1/0539/08 Slovak Grant Agency VEGA and APVV no. SK-SRB-0008-07.

** The research supported by Serbian Ministry of Science and Technological Development, Grant No. 144011. and by the Provincial Secretariat for Science and Technological Development, Autonomous Province of Vojvodina, Grant "Lattice methods and applications".
}

S. Chaudhury et al. (Eds.): PReMI 2009, LNCS 5909, pp. 116 2009.

(C) Springer-Verlag Berlin Heidelberg 2009 
called dominating classes. The best known are fuzzy partitions, arising from fuzzy equivalences (investigated by e.g., De Baets, Mesiar, Montes, Couso, Gil, 17/8], and by many others). For more papers about relational blocks, see references in the cited articles and books. Let us mention also the book 2 by Belohlavek, which provides a comprehensive introduction to fuzzy (lattice valued) relations.

In the present paper we deal with lattice-valued binary fuzzy relations. Due to our cutworthy approach, the co-domain of all fuzzy structures here is a complete lattice. Our aim is to investigate fuzzy blocks of an arbitrary fuzzy relation. Being fuzzy sets on the same domain, these blocks uniquely determine a family of subsets of the domain - its cuts. This family is a closure system under set inclusion.

In dealing with the opposite construction, we are motivated by the following real life problem. Suppose that each member of some group of persons is, by some preference, associated to several groups of other members of the same group. It is obviously a closure system on this group of people. Hence, there are as much closure systems on the group, as there are people in it. Now the problem is to determine a lattice, and a fuzzy (lattice valued) relation which connects all these closure systems. In addition, the cuts of the blocks of this relation should be the starting closure systems.

This problem is solved by the construction presented in the present article.

\section{Preliminaries}

If $\rho$ is a binary relation on a set $X, \rho \subseteq X^{2}$, then for $x \in X$ we denote by $\rho[x]$ the subset of $X$ defined by

$$
\rho[x]:=\{y \in X \mid(x, y) \in \rho\} .
$$

The set $\rho[x]$ is called the $x$-block of $\rho$.

The following lemma is straightforward.

Lemma 1. If $\rho=\bigcap_{i \in I} \rho_{i}$, then $\rho[x]=\bigcap_{i \in I} \rho_{i}[x]$.

A complete lattice is a partially ordered set $(L, \leq)$ whose every subset has a least upper bound (join, supremum) and a greatest lower bound (meet, infimum) under $\leq$. A complete lattice has the top and the bottom element, denoted respectively by 1 and 0 .

Lattices and related topics are presented e.g., in book [3].

A fuzzy set $\mu: X \rightarrow L$ is a mapping from a non-empty set $X$ (domain) into a complete lattice $L$ (co-domain). According to the original definition $L$ is the unit interval $[0,1]$ of real numbers (which is a complete lattice under $\leqslant$ ). However, we consider a complete lattice $L$ in a more general setting and sometimes we use the term $L$-fuzzy set, or lattice-valued (fuzzy) set 4 .

A mapping $R: X^{2} \rightarrow L$ (a fuzzy set on $X^{2}$ ) is a fuzzy ( $L$-fuzzy, latticevalued) relation on $X$.

If $\mu: X \rightarrow L$ is a fuzzy set on a set $X$ then for $p \in L$, the set

$$
\mu_{p}:=\{x \in X \mid \mu(x) \geqslant p\}
$$

is a $p$-cut, or a cut set, (cut) of $\mu$. More about cutworthy approach to latticevalued fuzzy structures can be found in 91011. 


\section{Results}

Let $R: X^{2} \rightarrow L$ be an $L$-valued binary relation on a set $X$. For every $x \in X$, the fuzzy $x$-block ( $x$-block) of $R$ is the $L$-valued set $R[x]: X \rightarrow L$, defined by

$$
R[x](y):=R(x, y), \text { for each } y \in X .
$$

Observe that we use the same name for the crisp $x$-block of a crisp relation (given by (11) above); still it is clear from the context which of these notions is used. We refer to blocks of $R$, meaning $x$-blocks, where $x$ runs over all $x \in X$. Being an $L$-valued set on $X$, every $x$-block of $R$ determines a collection of crisp subsets of $X$, its cuts.

Our main result is the following.

Theorem 1. Let $X$ be a nonempty set and for each $x \in X$ let $\mathcal{R}_{x}$ be a collection of nonempty subsets of $X$ closed under set intersection and containing $X$. Then there is a lattice $L$ and an $L$-valued relation $R$ on $X$, such that for every $x \in X$, $\mathcal{R}_{x}$ is the collection of nonempty cuts of a relational block $R[x]$.

First we describe a construction of the lattice $L$ and of the corresponding $L$ valued relation $R$, and then we prove the theorem by showing that the constructed relation fulfills the requirements.

Let $X$ be a nonempty set. By assumption of the theorem, for every $x \in X$,

$$
\mathcal{R}_{x}=\left\{U_{i}^{x} \mid i \in I\right\}
$$

is a family of subsets of $X$ which is closed under set intersection and which contains $X$ as a member. Observe that the cardinality of each family is the same, card $I$; in addition, some sets in the same family may be equal.

\section{The construction}

A Starting with $\mathcal{R}_{x}$, for each $x \in X$ define the following collection of subsets of $X^{2}$ :

$$
\overline{\mathcal{R}}_{x}:=\left\{\overline{U_{i}^{x}} \subseteq X^{2} \mid i \in I\right\} \text { where for every } i \in I, \overline{U_{i}^{x}}:=\left\{(x, u) \mid u \in U_{i}^{x}\right\} .
$$

In other words, $\overline{\mathcal{R}}_{x}$ is obtained in such a way that each element $u$ of every set in $\mathcal{R}_{x}$ is replaced by the ordered pair $(x, u)$.

B Construct a collection of ordinary (crisp) relations $\left\{\rho_{i} \mid i \in I\right\}$, so that for every $i \in I$

$$
\rho_{i}:=\bigcup_{x \in X}\left\{\overline{U_{i}^{x}} \mid \overline{U_{i}^{x}} \in \overline{\mathcal{R}}_{x}\right\} .
$$

C Construction of the lattice $L$ :

$$
L:=(\mathcal{C}, \leqslant),
$$

where $\mathcal{C}:=\left\{\widehat{\rho_{i} \mid i \in} I\right\}$, and the "hat" means that $\left\{\rho_{i} \mid i \in I\right\}$ is completed by adding to this collection all missing set intersections of its relations; the order $\leqslant$ is the dual of set inclusion 1

${ }^{1}$ In lattice-theoretic terms, Dedekind-McNeille completion of the poset $\left(\left\{\rho_{i} \mid i \in I\right\}, \subseteq\right)$ is constructed, and its dual is taken to be the lattice $L$. 
D Define the relation $R: X^{2} \rightarrow L$ by

$$
R(x, y):=\bigcap\{\rho \in \mathcal{C} \mid(x, y) \in \rho\}
$$

In the following lemma, we assume that all objects defined above, from $X$ to $\mathcal{C}$ and $R$ are given.

Lemma 2. Let $\rho \in \mathcal{C}$ and $x \in X$. Then the following holds:

(i) The $x$-block of $\rho$, if it is not empty, belongs to the family $\mathcal{R}_{x}$, namely: $\rho[x] \in \mathcal{R}_{x}$.

(ii) $\{u \in X \mid \bigcap\{\sigma \in \mathcal{C} \mid(x, u) \in \sigma\} \subseteq \rho\}=\rho[x]$.

Proof. (i) Observe that either $\rho=\rho_{i}$ for some $i \in I$, or $\rho=\bigcap\left\{\rho_{j} \mid j \in J \subseteq I\right\}$. In the first case,

$$
\begin{gathered}
\rho=\rho_{i}:=\bigcup_{x \in X}\left\{\overline{U_{i}^{x}} \mid \overline{U_{i}^{x}} \in \overline{\mathcal{R}}_{x}\right\} . \\
\rho_{i}[x]=\left\{u \in X \mid(x, u) \in \rho_{i}\right\} .
\end{gathered}
$$

Further, $\rho_{i}[x]=\left\{u \in X \mid(x, u) \in \overline{U_{i}^{x}}\right\}$.

$(x, u) \in \overline{U_{i}^{x}}$ is equivalent with $u \in U_{i}^{x}$.

Therefore,

$$
\rho_{i}[x]=U_{i}^{x}
$$

and $\rho[x]=\rho_{i}[x] \in \mathcal{R}_{x}$.

Now, suppose that $\rho=\bigcap\left\{\rho_{j} \mid j \in J \subseteq I\right\}$. By Lemma 1] $\rho[x]=\bigcap\left\{\rho_{j}[x] \mid j \in\right.$ $J \subseteq I\}$. Now we have, $\rho[x]=\bigcap\left\{U_{j}^{x} \mid j \in J \subseteq I\right\}$. Since, for every $x, \mathcal{R}_{x}$ is a family closed under intersection, then $\rho[x] \in \mathcal{R}_{x}$.

(ii) If $y \in\{u \in X \mid \bigcap\{\sigma \in \mathcal{C} \mid(x, u) \in \sigma\} \subseteq \rho$, then obviously $\bigcap\{\sigma \in \mathcal{C} \mid$ $(x, y) \in \sigma\} \subseteq \rho$, therefore $(x, y) \in \rho$, hence $y \in \rho[x]$. Conversely, if $y \in \rho[x]$, then $(x, y) \in \rho$, therefore $\rho$ belongs to the collection of relations that contain $(x, y)$, and thus $\bigcap\{\sigma \in \mathcal{C} \mid(x, y) \in \sigma\} \subseteq \rho$. Hence, $y \in\{u \in X \mid \bigcap\{\sigma \in \mathcal{C} \mid(x, u) \in$ $\sigma\} \subseteq \rho\}$.

Proof of Theorem [1. As defined by (2), $\{R[x] \mid x \in X\}$ is a collection of $L$-valued sets on $X$ which are the blocks of $R$; recall that for $x \in X$ and for every $y \in X$, we have $R[x](y)=R(x, y)$.

To prove the theorem, we show that for each $x \in X$, the collection of nonempty cuts of $R[x]$ coincides with the family $\mathcal{R}_{x}$ by which we started.

Let $x \in X$, and let $R[x]_{\rho}$ be the cut of the block $R[x]$, for some $\rho \in \mathcal{C}$. Now,

$R[x]_{\rho}=\{u \in X \mid R(x, u) \geqslant \rho\}=\{u \in X \mid R(x, u) \subseteq \rho\}=$

$\{u \in X \mid \bigcap\{\sigma \in \mathcal{C} \mid(x, u) \in \sigma\} \subseteq \rho\}=\rho[x] \in \mathcal{R}_{x}$,

by Lemma 2 (ii) and $(i)$, respectively.

Conversely, let $U_{i}^{x} \in \mathcal{R}_{x}$. By the fact (3) proved within Lemma $2(i), \rho_{i}[x]=$ $U_{i}^{x}$. By Lemma 2 ( $\left.i i\right)$,

$\rho_{i}[x]=\left\{u \in X \mid \bigcap\{\sigma \in \mathcal{C} \mid(x, u) \in \sigma\} \subseteq \rho_{i}\right\}=$

$\left\{u \in X \mid R(x, u) \geq \rho_{i}\right\}=R[x]_{\rho_{i}}$.

Hence $U_{i}^{x}=R[x]_{\rho_{i}}$. 
Example. Let $X=\{a, b, c\}$.

We start with three collections of subsets of $X$ which are closed under intersections:

$\{\{a\},\{b\},\{a, b\},\{b, c\},\{a, b, c\}\},\{\{a\},\{a, b\},\{a, b, c\}\},\{\{c\},\{a, b, c\}\}$.

In order to have families of the same cardinality (five members), we consider the sets in above collections to be ordered as they are listed, and we repeat the set $\{a, b, c\}$ necessary number of times (two and three times in the second and the third collection, respectively).

$$
\begin{aligned}
& \mathcal{R}_{a}=(\{a\},\{b\},\{a, b\},\{b, c\},\{a, b, c\}), \\
& \mathcal{R}_{b}=(\{a\},\{a, b\},\{a, b, c\},\{a, b, c\},\{a, b, c\}), \\
& \mathcal{R}_{c}=(\{c\},\{a, b, c\},\{a, b, c\},\{a, b, c\},\{a, b, c\}) .
\end{aligned}
$$

Relations $\overline{\mathcal{R}}_{a}, \overline{\mathcal{R}}_{b}$ and $\overline{\mathcal{R}}_{c}$, step $\mathbf{A}$ :

$$
\begin{aligned}
\overline{\mathcal{R}}_{a}= & (\{(a, a)\},\{(a, b)\},\{(a, a),(a, b)\},\{(a, b),(a, c)\},\{(a, a),(a, b),(a, c)\}) \\
\overline{\mathcal{R}}_{b}= & (\{(b, a)\},\{(b, a),(b, b)\},\{(b, a),(b, b),(b, c)\},\{(b, a),(b, b),(b, c)\}, \\
& \{(b, a),(b, b),(b, c)\}) \\
\overline{\mathcal{R}}_{c}= & (\{(c, c)\},\{(c, a),(c, b),(c, c)\},\{(c, a),(c, b),(c, c)\},\{(c, a),(c, b),(c, c)\},
\end{aligned}
$$

\begin{tabular}{|c|c|c|c|c|}
\hline 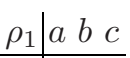 & $\rho_{2} \mid a b c$ & $\rho_{3} \mid a b c$ & $\rho_{4} \mid a b c$ & $\rho_{5} \mid a b c$ \\
\hline \begin{tabular}{l|llll}
$a$ & 1 & 0 &
\end{tabular} & \begin{tabular}{l|llll}
$a$ & 0 & 1 & 0
\end{tabular} & \begin{tabular}{l|lll}
$a$ & 1 & 1 & 0
\end{tabular} & \begin{tabular}{l|llll}
$a$ & 0 & 1 & 1
\end{tabular} & \begin{tabular}{l|lll}
$a$ & 1 & 1
\end{tabular} \\
\hline \begin{tabular}{l|lll}
$b$ & 1 & 0 & 0
\end{tabular} & \begin{tabular}{l|lll}
$b$ & 1 & 1 & 0
\end{tabular} & \begin{tabular}{l|lll}
$b$ & 1 & 1
\end{tabular} & \begin{tabular}{l|lll}
$b$ & 1 & 1
\end{tabular} & \begin{tabular}{l|lll}
$b$ & 1 & 1 & 1
\end{tabular} \\
\hline \begin{tabular}{l|llll}
$c$ & 0 & 0 & 1
\end{tabular} & \begin{tabular}{l|ll}
$c$ & 111
\end{tabular} & \begin{tabular}{l|ll}
$c$ & 111
\end{tabular} & \begin{tabular}{l|ll}
$c$ & 111
\end{tabular} & $\begin{array}{llll}c & 111\end{array}$ \\
\hline
\end{tabular}
$\{(c, a),(c, b),(c, c)\})$.

Next we construct relations $\rho_{1}, \ldots, \rho_{5}$, as defined in step B: $\rho_{1}=\{(a, a)\} \cup$ $\{(b, a)\} \cup\{(c, c)\} ; \rho_{2}=\{(a, b)\} \cup\{(b, a),(b, b)\} \cup\{(c, a),(c, b),(c, c)\} ; \quad$ etc. :

Now the missing set intersections of the above relations:

$$
\begin{aligned}
& \rho_{6}=\rho_{1} \cap \rho_{2} ; \quad \rho_{7}=\rho_{3} \cap \rho_{4}
\end{aligned}
$$

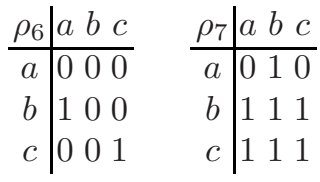

$\mathcal{C}=\left\{\rho_{1}, \ldots, \rho_{7}\right\}$, the lattice $L$ (step $\mathbf{C}$ ) is in Figure 1 .

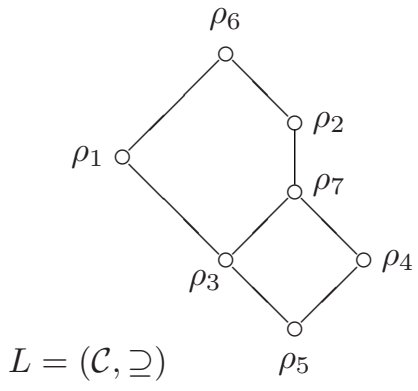

Fig. 1. 
Construction of fuzzy relation $R: X^{2} \rightarrow L$ (step D):

$R(a, a)=\bigcap\{\rho \in \mathcal{C} \mid(a, a) \in \rho\}=\rho_{1} \cap \rho_{3} \cap \rho_{5}=\rho_{1} ;$

$R(b, c)=\bigcap\{\rho \in \mathcal{C} \mid(b, c) \in \rho\}=\rho_{3} \cap \rho_{4} \cap \rho_{5} \cap \rho_{7}=\rho_{7}$; etc. :

$$
\begin{array}{c|ccc}
R & a & b & c \\
\hline a & \rho_{1} & \rho_{2} & \rho_{4} \\
b & \rho_{6} & \rho_{2} & \rho_{7} \\
c & \rho_{2} & \rho_{2} & \rho_{6}
\end{array} .
$$

The blocks of $R$ are defined by the rows of the above table:

$$
R[a]=\left(\begin{array}{ccc}
a & b & c \\
\rho_{1} & \rho_{2} & \rho_{4}
\end{array}\right),
$$

and the corresponding cut sets are: $R[a]_{\rho_{1}}=\{a\}, R[a]_{\rho_{2}}=R[a]_{\rho_{7}}=\{b\}$, $R[a]_{\rho_{3}}=\{a, b\}, R[a]_{\rho_{4}}=\{b, c\}, R[a]_{\rho_{5}}=\{a, b, c\}, R[a]_{\rho_{6}}=\emptyset$. Hence, the nonempty cuts of $R[a]$ are the sets in $\mathcal{R}_{a}$.

Analogously, one can check that the collection of nonempty sets in $R[b]$ coincides with the family $\mathcal{R}_{b}$; the same holds for the cuts in $R[c]$ and the sets in $\mathcal{R}_{c}$.

\section{Conclusion}

We have solved the problem of the construction of a relation, if collections of cuts of it blocks are given. As mentioned, this construction could be widely applied. Our next task is to investigate properties which should be fulfilled by the given closure systems, in order that the obtained fuzzy relation possesses some particular properties, like reflexivity, symmetry, antisymmetry, transitivity etc.

\section{References}

1. De Baets, B., Mesiar, R.: T-partitions. Fuzzy Sets and Systems 97, 211-223 (1998)

2. Bělohlávek, R. (ed.): Fuzzy Relational Systems: Foundations and Principles. Kluwer Academic/Plenum Publishers, New York (2002)

3. Davey, B.A., Priestley, H.A.: Introduction to Lattices and Order. Cambridge University Press, Cambridge (1992)

4. Goguen, J.A.: L-fuzzy Sets. J. Math. Anal. Appl. 18, 145-174 (1967)

5. Gottwald, S.: Fuzzy sets and Fuzzy Logic. Vieweg (1993)

6. Klir, G., Yuan, B.: Fuzzy sets and fuzzy logic. Prentice-Hall, Englewood Cliffs (1995)

7. Montes, S., Couso, I., Gil, P.: Fuzzy $\delta-\varepsilon$-partition. Information Sciences 152, 267-285 (2003)

8. Murali, V.: Fuzzy equivalence relations. Fuzzy Sets and Systems 30, 155-163 (1989)

9. Šeselja, B., Tepavčević, A.: Representing Ordered Structures by Fuzzy Sets, An Overview. Fuzzy Sets and Systems 136, 21-39 (2003)

10. Šešelja, B., Tepavčević, A.: Completion of ordered structures by cuts of fuzzy sets: An overview. Fuzzy Sets and Systems 136, 1-19 (2003)

11. Tepavčević, A., Vujić, A.: On an application of fuzzy relations in biogeography. Information Sciences 89(1-2), 77-94 (1996) 